\title{
2015 Steele Prizes
}

The 2015 Leroy P. Steele Prizes were presented at the 121st Annual Meeting of the AMS in San Antonio, Texas, in January 2015. The Steele Prizes were awarded to ROBERT LAZARSFELD for Mathematical Exposition; to ROSTISLAV GRIGORCHUK for Seminal Contribution to Research; and to VICTOR KAC for Lifetime Achievement.

\section{Mathematical Exposition: Robert Lazarsfeld}

\section{Citation}

The 2015 Leroy P. Steele Prize for Mathematical Exposition is awarded to Robert Lazarsfeld for his books Positivity in Algebraic Geometry I Ergebnisse der Mathematik und iher Grenzgebiete. 3. Folge. A Series of Modern Surveys in Mathematics, 48, Springer-Verlag, Berlin, 2004) and Positivity in Algebraic Geometry II (Ergebnisse der Mathematik und iher Grenzgebiete. 3. Folge. A Series of Modern Surveys in Mathematics, 49, Springer-Verlag, Berlin, 2004).

Lazarsfeld's books Positivity in Algebraic Geometry I and II were instant classics. He has succeeded wonderfully in putting together under the same heading most of the areas of classical and modern complex algebraic geometry dedicated to, or influenced by, the study of positivity. The positivity of the title encompasses a circle of ideas germinating from the notion of an ample line bundle on a smooth projective variety. Roughly speaking, such a line bundle induces an embedding of the variety into a projective space. By means of this embedding, the restriction of the classical Fubini-Study metric gives rise to a positive, or Kähler, form on the variety in the sense of differential geometry. Fundamental work of Kodaira showed that, conversely, positivity in the differential-geometric sense implies positivity in the algebro-geometric one. This circle of ideas has been enlarged over the years to include singular varieties, higher rank bundles, and eventually more general sheaves, singular Kähler metrics, and fractional divisors, leading to the introduction of multiplier ideals in both analytic and algebraic settings. Here, for the first time in textbook form, the resulting algebraic theory of multiplier ideals is fully developed. Furthermore, Lazarsfeld's books work out the theory of asymptotic multiplier ideals, tools that have since

DOI: http://dx.doi.org/10.1090/noti1231 become of utmost importance in birational geometry; they were used in the proofs of landmark theorems such as the invariance of plurigenera, the finite generation of the canonical ring, and the existence of flips. His books are now used for courses and for reading and research seminars all over the world. They can be used both as textbooks and as sources for current research problems, and so have great value for both students and experts in the field. They are exceptionally well written, with numerous examples, new proofs, and especially new results reflecting Lazarsfeld's great taste and originality.

\section{Biographical Sketch}

Robert Lazarsfeld was born in New York City in 1953. He graduated from Harvard College in 1975 and received his $\mathrm{PhD}$ from Brown University in 1980 under the direction of William Fulton. After a postdoctoral stint as an instructor at Harvard, he went to the University of California Los Angeles in 1983. He remained there until moving to the University of Michigan in 1997, where in 2007 he became the Raymond L. Wilder Collegiate Professor. Since 2013, Lazarsfeld has been on the faculty of the mathematics department at Stony Brook University.

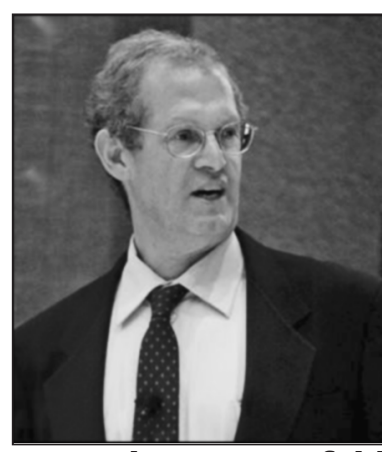

Robert Lazarsfeld
Lazarsfeld was

a Sloan Foundation Fellow (1984), a Presidential Young Investigator (1985-1990), an invited speaker at the International Congress of Mathematicians (1990), and a Guggenheim Fellow (1998). He delivered the 2005 AMS Colloquium Lectures and was elected to the American Academy of Arts and Sciences in 2006. From 2002 to 2009, Lazarsfeld served on the editorial board of the Journal of the American Mathematical Society, and in 2012 he became a Fellow of the AMS.

\section{Response from Robert Lazarsfeld}

I am delighted and honored to receive the 2015 Leroy P. Steele Prize for Mathematical Exposition for my monograph on positivity in algebraic geometry. Writing it was a protracted labor of love-or, as it occasionally seemed, madness-so naturally 
I'm very gratified that in the end the book appears to have proved itself useful. I've always believed that it's worth striving to present mathematics well, and I'm pleased to have these efforts acknowledged here.

The book owes a great deal to many teachers, students, and colleagues, but I'd like to single out three to whom I have a particular debt. To begin with I'm grateful to János Kollár for suggesting in the first place that I might write a book for the Ergebnisse series. I am also indebted to Bill Fulton, who not only introduced me as a graduate student to the circle of questions around positivity but also taught me the importance of good mathematical writing. Finally, quite a bit of the material that appears in the book I either learned from Lawrence Ein or worked out together with him, and I'd like to take this opportunity to express my thanks to him.

\section{Seminal Contribution to Research: Rostislav Grigorchuk}

\section{Citation}

The 2015 Leroy P. Steele Prize for Seminal Contribution to Research is awarded to Rostislav Grigorchuk for his article "Degrees of growth of finitely generated groups and the theory of invariant means", Izvestiya Akademii Nauk SSSR. Seriya Matematicheskaya, 48 (1984), no. 5, 939-985. In this paper Grigorchuk constructed the first examples of a finitely generated group whose growth was faster than polynomial but slower than exponential, answering a question raised by John Milnor in 1968. These groups had other amazing properties: they are torsion, they give a new counterexample to the Burnside problem, and they are amenable, but not elementary amenable, thus answering another old question of Mahlon Day. Moreover, Grigorchuk's groups turned out to be just infinite and yet have finite nilpotent width.

Grigorchuk showed that the class of groups of intermediate growth is extremely rich: it contains a continuum of 2-generator pairwise distinct groups with respect to quasi-isometry, a relation much weaker than isomorphism of groups. This breakthrough greatly sharpened results of $\mathrm{B}$. $\mathrm{H}$. Neumann, who had proved the existence of a continuum of nonisomorphic 2-generator groups in 1937.

It is important that Grigorchuk's work not only gave solutions to old standing problems but also discovered new exciting classes of groups which became known as "branch groups" and "selfsimilar groups". These groups provided examples with the previously unknown asymptotic behavior of random walks and the spectral densities of discrete Laplacians. They found applications in the theory of fractals, holomorphic dynamics, spectral theory of groups and graphs, and theory of finite automata.
Grigorchuk used the idea of Kolmogorov complexity to study the complexity of algorithmic problems in his groups. This work attracted the attention of computer scientists and cryptographers.

The work of Grigorchuk has influenced several generations of researchers in group theory. It would be impossible to imagine modern group theory without Grigorchuk's work.

\section{Biographical Sketch}

Rostislav Grigorchuk is Distinguished Professor at Texas A\&M University. He received his PhD from Lomonosov Moscow State University in 1978 , advised by Dmitry Anosov and Anatoly Stepin. He taught from 1978 to 1995 at the Moscow State University of Railway Engineering, Rostislav Grigorchuk where he also eventually occupied the position of head of the Department of Mathematics, and from 2000 to 2002 at Lomonosov Moscow State University. He was invited to the Steklov Institute of Mathematics (Moscow) in 1995 and worked there as a leading researcher from 1995 to 2002. In 2002 he moved to College Station, Texas, in the United States.

Grigorchuk was born in 1953 in the small village of Mukhavets in the Vishnivci district of the Ternopilska region in Ukraine. He received the Prize of the Moscow Mathematical Society in 1979, was an invited speaker at the International Congress of Mathematicians in Kyoto (1990), was a Fulbright Foundation Senior Fellow affiliated with Columbia University in 1991 (by invitation of Hyman Bass), twice received the Award for Best Scientific Results from the Russian Academy of Science (1999 and 2001), received the Distinguished Achievement Award from the Association of Former Students of Texas A\&M University (2009), and was honored as a Fellow of the AMS in 2013.

His research interests are in group theory, dynamical systems, random walks, spectral theory of groups and graphs, operator algebras, theory of fractals, theory of automata, and formal languages.

\section{Response from Rostislav Grigorchuk}

It is a great honor to receive this award. I credit my father, Ivan Grygorchuk, and my high school math teachers, Vira Habryd in Ivanovo-Frankivsk and Volodymyr Axelrod in Chernivtci (Ukraine), for stimulating my interest in mathematics and teaching me how to achieve my goals. From my thesis adviser Anatoly Stepin at Moscow University and my further mentors Dmitri Anosov and Yakov Sinai, I learned many things, in particular how to mix ergodic theory with algebra and functional analysis and not to be afraid to attack difficult 
problems. It was a pleasure listening to a talk in one of their seminars for three to four hours (for instance, a talk of G. Kasparov or M. Lyubich) and then to play soccer the next day against the team of another seminar. I furthermore learned a lot from Sergei Adian and Vladimir Arnold, to whom I am also grateful.

Group theory is a fascinating area of mathematics with a prominent past and future. Infinite finitely generated groups and their approximation by finite groups play a special role in various contexts. Such great problems of modern mathematics as the Baum-Connes conjecture, the Novikov conjecture, and the Atiyah conjectures are in equal measure problems in topology and group theory. The same is true for the Poincaré and AndrewsCurtis conjectures.

The developments of the last few decades, including studies around the Burnside problem (P. Novikov, S. Adian, E. Golod, E. Zelmanov), the theory of hyperbolic groups initiated by Gromov, groups with automatic structure as introduced by Cannon and Thurston, groups of intermediate growth as discovered following Milnor's question about their existence, the notion of amenability invented by von Neumann and N. Bogolyubov, property (T) of Kazhdan, and much more, have enriched group theory, led to powerful applications, and made it an attractive area for young researchers. The Leroy P. Steele Prize for Seminal Contribution to Research that is awarded to me is a reflection of the growing authority and popularity of group theory.

I thank my students, collaborators, and colleagues for their support, patience, and interest in my research and my lectures. I am grateful to the administration of my department and of the College of Science at Texas A\&M University, where I enjoy excellent conditions for teaching and carrying out research. My wife Nina, my daughter Olesya, and my parents were always very supportive in my work and shared with me the difficulties and pleasures that life serves to us. To all of them, and to the Steele Prize Committee and the American Mathematical Society, I express my deepest gratitude.

\section{Lifetime Achievement: Victor Kac \\ Citation}

The structure and representation theory of finitedimensional Lie groups and Lie algebras has left no part of mathematics untouched since the mid1800s. The search for an analogous theory for infinite-dimensional Lie groups and algebras is much more recent but is no less important and fundamental. Victor Kac has been the prime mover in creating this theory over the past forty-five years.

Kac-Moody algebras, introduced by Kac and Moody in 1967, quickly became a basic and natural area of investigation in representation theory.
They now are seen to form the backbone of many aspects of combinatorics, integrable systems, modular forms, enumerative algebraic geometry, and the Langlands program. They also play an important role in quantum field theory and statistical mechanics. Kac's discovery of the Weyl-Kac character formula laid the foundation for the study of representation theory of Kac-Moody algebras and yielded the interpretation of the famous Macdonald identities as denominator identities. Kac's work on the representation theory of the Virasoro algebra has found important applications to integrable systems, conformal field theory, statistical physics, and the study of moduli spaces of curves.

Kac's classification of simple Lie superalgebras, echoing the nineteenth-century Killing-Cartan classification of simple Lie algebras, has had an enormous influence in setting the stage for subsequent developments in super geometry. Kac's theorems and conjectures on the representation theory of quivers have shaped the development of this growing field.

Kac's recognition theorem and the Kac-Weisfeiler conjectures paved the way to a much deeper understanding of the structure and representations of modular Lie algebras. Recently, Kac and his students developed structure and representation theories of linearly compact superalgebras and related theories of conformal algebras and pseudoalgebras.

Several generations of mathematicians have found the three editions of Kac's book InfiniteDimensional Lie Algebras (third edition, Cambridge University Press, Cambridge, 1990) indispensable to their education and research.

The 2015 Leroy P. Steele Prize for Lifetime Achievement is awarded to Victor Kac in recognition of all these achievements.

\section{Biographical Sketch}

My ancestors lived in a small Romanian shtetl called Lipcani. My father grew up in a wealthy family, and after high school he was supposed to go to Paris to study at the Sorbonne. Instead, he chose to stay in Lipcani for three more years waiting for his beautiful sweetheart to graduate from high school. In 1940, my future

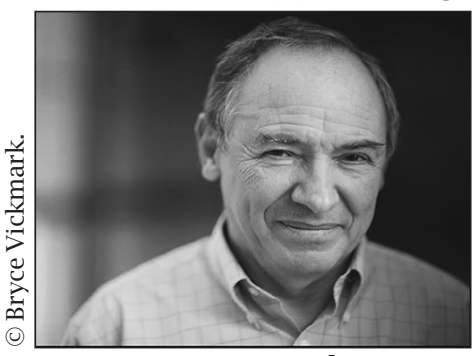
parents enrolled in Kishinev University. Soon after, the eastern part of Romania was annexed by the Soviet Union, and the wealthiest citizens of Lipcani, including my paternal grandparents, were arrested and deported to Siberia.

A year later, Germany invaded the Soviet Union, and Romanian forces, working with the German 
fascists, rounded up all the Jews from Lipcani and sent them to their deaths in concentration camps. Thus, all my great-grandparents, my maternal grandparents, and many other members of our extended family perished in the Holocaust. Luckily, my parents were evacuated with the rest of the students at Kishinev University to the Ural Mountains, where I was born on December 19, 1943.

In 1944 we returned to the liberated Kishinev. My parents found jobs in a publishing house; they worked very long hours to earn a living. To this day I remember the clicking of the typewriter every evening as I was going to bed and every morning as I was waking up.

I fondly remember the Kishinev school where I studied from 1950 to 1960 . In the summer of 1959, a friend from high school persuaded me to participate in a local Math Olympiad, where, to my astonishment, I won second place. It was then that I decided to become a mathematician. I went to a nearby library and took out all available books with math problems. At first, it would take me several days to solve a problem, but one by one I mastered them all. Encouraged, I enrolled in the Math Circle at Kishinev University. There I met a girl who told me that she was not going to study in our provincial university but would go to Moscow instead. I instantly decided that I too would try to get accepted into Moscow University.

The years 1960-1968 at Moscow University were the happiest of my "Soviet" life. How can one describe in a few lines the excitement and the enthusiasm of those years? The university was the center of mathematics and independent thinking in a huge country - a powerful fountain of new ideas and the source of most important discoveries.

During my sophomore year, I was incredibly fortunate to meet Ernest Vinberg, then still a graduate student, who, together with Arkadii Onishchik, had just founded a Lie group seminar, an offshoot of the famous Dynkin seminar. (By this time Eugene Dynkin had turned his full attention to probability.) Two years later Vinberg gave me a problem, which determined the direction of my research for the rest of my life. Under his guidance, in 1965 I defended my master's thesis "Algebraic definition of compact Lie groups" and my $\mathrm{PhD}$ thesis "Simple irreducible graded Lie algebras of finite growth" in 1968. I admire mathematicians who change the direction of their research several times, each time starting from scratch. This has never happened in my case, where each new direction was a natural offshoot of my previous work.

After graduating, I was able to find a job at the Moscow Institute of Electronic Machine Building, a school with no graduate program and a very large teaching load. There wasn't even a slightest chance for me to find another job, as new draconian anti-Semitic practices had been introduced in the country. Even worse, most Soviet math journals would not publish my papers, and it was next to impossible to obtain permission to publish abroad.

Fortunately, in the beginning of the 1970s a new beacon of hope brightened the grim Soviet reality: a mass emigration had begun. When my friends David Kazhdan and Boris Weisfeiler emigrated, it came as a shock, but gradually I understood that I too had no other choice. In 1977 I left the Soviet Union.

While in Rome waiting for a U.S. visa, I was offered a job at MIT, and on March 15, 1977-the date which I still consider the most important of my life-I arrived in the United States. The following day I came to MIT. Without even asking for my documents, a department administrator gave me a key to my office and asked for my bank account number. "What is a bank account?" I asked. My new life had begun.

\section{Response from Victor Kac}

It is a great honor to be awarded the 2015 Leroy P. Steele Prize for Lifetime Achievement. I am humbled to be among so many great mathematicians awarded the prize in previous years. I am most grateful to the American Mathematical Society for this prestigious award, and particularly to the members of the Steele Prize Committee for making this difficult decision.

I would like to credit my mentors for their support:

- Ernest Vinberg, who was my thesis advisor at Moscow University, and to whom I've been always turning when getting stuck in a problem.

- Bert Kostant, who convinced his colleagues to grant tenure to a hapless refugee from the Soviet Union.

- Is Singer and Eugene Dynkin, who gave me crucial support in a difficult period of my "western" life.

- David Kazhdan, who, being a few years younger than me, was nevertheless my mentor with his infinite knowledge and wisdom.

I've been blessed in having many collaborators. It is a pleasure to acknowledge them-over sixtyfive in number. It is impossible to list all of them here and the work we have done. However, I must mention the three men of immense mathematical talent with whom I coauthored more than ten papers:

- Dale Peterson: Together we discovered modular invariance of characters of affine Lie algebras and the "Kac-Peterson class", and we built the foundations of the theory of Kac-Moody groups.

- Minoru Wakimoto: Together we discovered the "Kac-Wakimoto spectrum" (as called nowadays by physicists); we made advances in representation theory of affine Lie superalgebras and its connections to representation theory of superconformal algebras via the quantum Hamiltonian reduction; and we made advances in number 


\section{4 \\ CMS \\ $\overline{\mathrm{SMC}}$}
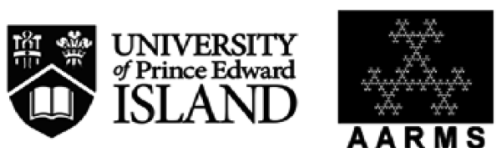

The Canadian Mathematical Society (CMS), the University of Prince Edward Island (UPEI) and the Atlantic Association for Research in the Mathematical Science (AARMS) invite the math community to the...

\section{CMS Summer Meeting}

Site: UPEI, Charlottetown, P.E.I., Canada June 5-8, 2015

Scientific Directors:

Gordon MacDonald, Shannon Fitzpatrick (UPEI)

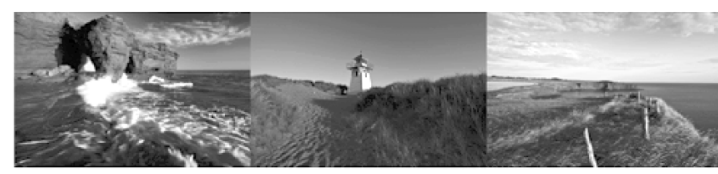

PRIZE LECTURES

CMS Coxeter-James Prize

CMS Excellence in Teaching Award

CMS Krieger-Nelson Prize

PRIZE LECTURE

Richard Nowakowski (Dalhousie)

PLENARY LECTURES

Nilima Nigam (SFU)

Christiane Rousseau (Montréal)

James Yorke (Maryland)

EOMCATION PLENARY LECTURE

Robert Ghrist (Pennsylvania)

\section{HIGHLIGHTS}

AARMS-CMS Student Poster Session

Contributed Papers

Education Session

More than 15 Scientific Sessions

CMS Annual General Meeting

CMS Awards Banquet with Lobster Supper

Please see our website for details: cms.math.ca/events/summer15

\section{Experience Charlottetown}

theory, especially to the emerging theory of mock theta functions.

- Alberto De Sole: Together with our students, we've been building the foundations of rigorous algebraic theory of infinite-dimensional integrable systems.

I should also mention the joint work with Corrado De Concini and Claudio Procesi on quantum groups at roots of unity and the work with them and Enrico Arbarello relating representation theory to the geometry of algebraic curves. We retained our friendship throughout the years since my emigration period in Rome, when I received their helping hand.

\section{About the Prize}

The Steele Prizes were established in 1970 in honor of George David Birkhoff, William Fogg Osgood, and William Caspar Graustein. Osgood was president of the AMS during 1905-1906, and Birkhoff served in that capacity during 1925-1926. The prizes are endowed under the terms of a bequest from Leroy P. Steele. Up to three prizes are awarded each year in the following categories: (1) Lifetime Achievement: for the cumulative influence of the total mathematical work of the recipient, high level of research over a period of time, particular influence on the development of a field, and influence on mathematics through $\mathrm{PhD}$ students; (2) Mathematical Exposition: for a book or substantial survey or expository research paper; (3) Seminal Contribution to Research: for a paper, whether recent or not, that has proved to be of fundamental or lasting importance in its field or a model of important research. Each Steele Prize carries a cash award of US $\$ 5,000$.

The list of previous recipients of the Steele Prize may be found on the AMS website at www. ams. org/prizes-awards

Beginning with the 1994 prize, there has been a five-year cycle of fields for the Seminal Contribution to Research Award. The 2015 prize was given in the field of algebra. The 2016 prize will be given in applied mathematics; the 2017 prize in geometry/topology; the 2018 prize in discrete mathematics (logic alternates with discrete mathematics every five years); the 2019 prize in analysis; and the 2020 prize in algebra, renewing the cycle. 